\title{
¿Qué tan riesgosas son las Cajas Municipales en el perú?
}

\author{
Mg. Econ. Renzo GAMBETTA PODESTÁ ${ }^{11}$
}

\section{RESUMEN:}

Para el presente artículo se utilizan técnicas de simulación de Monte Carlo para calcular la distribución de las pérdidas observadas en las carteras de préstamos durante 2013 y 2014 para cada Caja Municipal. Ratios de capital regulatorio se comparan con aquellos calculados en la simulación, con el objetivo de verificar los niveles de solvencia. Asimismo las cuentas de resultados se utilizan para lograr una medida de rentabilidad ajustada al verdadero riesgo que enfrentas las Cajas. El análisis se llevo a cabo con información del reporte regulatorio llamado RCD (Reporte Crediticio de Deudores) y que utiliza la SBS (Superintendencia de Banca y Seguro) para monitorear los riesgos crediticios del sistema financiero. Los resultados muestran que los ratios de rentabilidad contables difieren de los calculados en el ejercicio debido a que no se estaría tomando en cuenta los riesgos reales que enfrentan para lograr tales retornos. En términos de solvencia, el resultado es mixto, el requerimiento de capital regulatorio por riesgo de crédito en alguna Cajas estaría subestimada incluso no estarían cubriendo el mínimo legal regulatorio del diez por ciento (10\%).

PALABRAS CLAVE: Caja Municipal de Ahorro y Crédito, distribución de perdidas, prestamos, riesgos

\section{ABSTRACT:}

The article uses a resampling based on Monte Carlo simulation techniques to calculate distribution for the losses observed in the loans portfolios during 2013 and 2014 for each Caja Municipal. Regulatory capital ratios are compared with the unexpected losses to verify levels of solvency. Likewise the income statements are used to achieve a differently measure of the commons accountant financial profitability ratios for better allocation to the adjusted returns of credit risk of each Caja Municipal. The analysis was conducted with information from RCD (Reporte Crediticio de Deudores), regulatory report submitted for the SBS (Superintendencia de Banca y Seguros). The results show that the simple profitability ratios differ from those calculated in the simulation because they 11 Comentarios y/o sugerencias a regpo77@hotmail.com org04@cema.edu.ar 
would not take into account the real risks they face to achieve such returns. In terms of solvency the result is mixed, the regulatory capital requirement for credit risk in some Cajas Municipales would be underestimated even they would not be covering the legal minimum of ten percent $(10 \%)$.

KEY WORDS: Municipal Savings and Credit, Loss Distribution, Loans, Risk

\section{INTRODUCCIÓN}

Las Cajas Municipales de Ahorro y Crédito (CMAC) a lo largo de sus más de 30 años de creación han logrado ubicarse como las instituciones expertas en el sector de las micro finanzas, con el objetivo principal de desarrollar la actividad económica de las micro y pequeñas empresas (MYPE), así como el de lograr la inclusión financiera de más peruanos de los sectores socioeconómicos más bajos del país. El Sistema de Cajas Municipales constituye una alternativa financiera para atender las necesidades de los sectores de menores recursos, contribuyendo a hacer realidad sus emprendimientos y, con ello, dinamizar la economía y contribuir a disminuir la pobreza local ${ }^{12}$. Para su sano desarrollo y crecimiento las CMAC's deben de generar utilidades, constituir reservas para reforzar sus patrimonios y realizar obra social. Esto último incorpora un riesgo adicional en el sentido de que pueden ser utilizadas de manera populista ya que a pesar de que su objetivo principal es del tipo social, pueden verse incentivadas a actuar como instituciones "puramente privadas", es decir, incrementar el apetito de riesgo pudiendo poner en peligro la solvencia de la institución por la búsqueda de mayores retornos acumulando mayores fondos disponibles para la administración municipal en curso y dejando en segundo plano el fortalecimiento de sus patrimonios. Al cierre del año 2014 las CMACs alcanzaron los S/. 13,438 millones en créditos directos así como S/. 13,305 millones en depósitos totales, lo que representó un incremento interanual de $8 \%$ y $4 \%$ respectivamente. Al cierre del 2014, los créditos directos de las CMACs representaron el 7\% de los créditos de la banca múltiple y el $11 \%$ en términos de depósitos, cuando en el 2010 la participación en depósitos era de 7\% y en créditos directos de $8 \%$, mostrando esto la fuerte competencia que sufrieron por parte de los bancos en los segmentos donde mayormente operan las Cajas que son los créditos destinados a la micro y pequeña empresas.

El ratio de capital global (Patrimonio efectivo/ Activos ponderados por riesgo) reportado de las CMACs al 2010 era de 16.8\%, al cierre del 2014 los ratios llegaron a $14.51 \%$, lo que muestra una caída de -228 puntos básicos. Si bien son ratios que se ubican por encima del regulatorio (10\%), las Cajas han reducido sus niveles de solvencia y ante la necesidad de incrementar los niveles de rentabilidad, las Cajas vienen mostrando incrementos en los niveles de riesgo a través de mayores ratios de morosidad que podría deberse a menores restricciones en los

12 www.fpcmac.org.pe/files/librofepcmac.pdf 
procedimientos de otorgamiento de créditos así como al incentivo en elevar los tamaños de los prestamos promedio orientados a segmentos que no les son tan familiares y por lo tanto carecen del expertise ${ }^{13}$ necesario. Las mejores prácticas definidas en Basilea II en riesgo de crédito y el consenso mundial, exigen a las instituciones financieras cubrir las pérdidas medias o esperadas a través de las provisiones y las no esperadas a través del capital. El presente artículo va en ese sentido, utilizando la metodología de bootstrapping ${ }^{14}$ se estiman las diferentes distribuciones de pérdidas en los portafolios de crédito de cada una de las Cajas Municipales de manera empírica. Esta metodología no paramétrica no asume ningún tipo de distribución a priori de las pérdidas sino que permite replicar de manera fehaciente la distribución que realmente genero los datos de pérdidas minimizando los impactos en errores de medición. Los modelos más conocidos en la industria como son KMV, Credit Risk $+{ }^{15}$ y el propuesto por Basilea asumen supuestos específicos que podrían diferir de lo que ocurre realmente con las pérdidas empíricas.

\section{METODOLOGÍA}

Se realizaron dos ondas de análisis; La primera con todos aquellos clientes que a enero de 2012 tenían menos de 90 días de atraso en sus créditos consolidados realizando el seguimiento a partir de dicha fecha mes a mes por los siguientes 12 meses. En caso el cliente hubiera tenido durante dichos meses algún incumplimiento mayor a 90 días se le consideró un cliente en default, caso contrario el cliente pago adecuadamente sus obligaciones. Para la segunda onda se utilizó la misma metodología, salvo que el comienzo de la fecha de análisis fue enero de 2013. Todos aquellos clientes que tuvieron moras mayor o igual a 90 días se les asignó una severidad de pérdida (loss given default) del 75\% del saldo original. Suponemos que hay una tasa de recuperación optimista del $25 \%$ en función al porcentaje cubierto promedio en ambos años analizados por garantías preferidas que llegaron a representar el $20 \%$ del total de créditos directos según información de la SBS. A los 12 meses de observación se identifican todos aquellos clientes que tuvieron moras máximas de más de 90 días, por lo que son clasificados en 2 estados absorbentes;

$$
X= \begin{cases}1 & \text { si el deudor cumplió con la condición de default } \\ 0 & \text { si el deudor no cumplió con la condición de default }\end{cases}
$$

13 La gestión de riesgo en todo su ciclo (originación, monitoreo, cobranza y recuperación) difiere entre créditos destinados a las microempresas del resto, como pueden ser; créditos al consumo, hipotecas y créditos para empresas de mayor tamaño y ciclos más complejos.

14 El bootstrap es un método de remuestreo propuesto por Bradley Efron en 1979. Sirve para aproximar la distribución en el muestreo de un estadístico con el objetivo de verificar sesgo y variabilidad en un análisis estadístico, construir intervalos de confianza o realizar contrastes de hipótesis.

15 www.moodysanalytics.com/About-Us/History/KMV-History. 
El objetivo es poder contar con un grupo estadísticamente grande de muestras que serán aleatorias y muy semejantes unas de otras para poder definir los parámetros buscados de la distribución empírica de los datos. Para cada institución se extrajeron entre 500 y 700 deudores de manera aleatoria sin reemplazos y según el muestreo óptimo simple aleatorio para proporciones con un nivel de confianza del 99\% y un error del 3\%. Seguidamente, se repitió 20,000 veces este proceso con reemplazos y de forma aleatoria. Con estos parámetros nos aseguramos adecuados intervalos de confianza para métodos del tipo bootstrap. Para cada portafolio se computaron las pérdidas como porcentaje del valor inicial vigente. Para esto se realizó el cálculo sobre todos aquellos clientes considerados como "malos" ajustados por el factor de severidad de la pérdida sobre la deuda vigente a comienzos de cada año. Para el caso de las pérdidas esperadas (expected losses) se utilizaron las pérdidas medias de los 20,000 subportafolios nuevos y para las pérdidas inesperadas (unexpected losses) la diferencia entre VaR elegido (al 99.9\%) y la pérdida esperada. La metodología nos permitió construir la distribución de probabilidad empírica a partir de las diferentes muestras replicadas, asignando una probabilidad de $1 / \mathrm{n}$ a cada punto (misma probabilidad de selección) $x_{1}, x_{2}, x_{3}, x_{4}, \ldots \ldots x_{n}$ donde $n$ es el número óptimo de muestras. Como resultado obtenemos una función empírica de la variable $X$, siendo este el estimador no paramétrico de máxima verosimilitud de la función poblacional. Con la función empírica calculada extraemos muestras aleatorias simples para luego calcular los estadísticos de interés. El paso anterior se repite $Y$ veces, donde $Y$ es el número de subportafolios, que en este caso corresponde a 20,000.

$$
\text { Es decir; } \widehat{\varnothing}=\frac{\sum_{n=1}^{500 / 700} \text { Perdıdas }}{\sum_{n=1}^{500 / 700} \text {.Saldo Inicial }}
$$

Siendo $\widehat{\emptyset}$ el estimador bootstrapping del parámetro $\emptyset$ definido como la media del valor del estadístico calculado en las $\mathrm{n}$ deudores. Definición de Valor en Riesgo (VaR); Sea X la variable aleatoria que representa la pérdida en un portafolio de crédito. En función a dicha distribución y bajo un nivel de confianza definido $\alpha$, el VaR y el CVaR del portafolio se calculan de la siguiente manera:

$$
\operatorname{VaR}_{\alpha}=\inf \{x \mid P[X>x] \leq 1-\alpha\} \quad y \text { el } \quad C V a R_{\alpha}=E(L \mid L) \operatorname{VaR} R_{\alpha}=\frac{E\left(L, L \geq V a R_{\alpha}\right)}{P\left(L \geq V a R_{\alpha}\right)}
$$

\section{RESULTADOS}

El objetivo del articulo más que analizar las formas de distribución de las pérdidas es la identificación de las medidas más utilizadas para la cuantificación del riesgo de crédito; la pérdida esperada (Expected losses) y el Valor en Riesgo (VaR al 99.9\%). Adicionalmente y desde el punto de vista referencial, también se calculo el CVaR (Conditional Value at risk) como una medida de mayor sen- 
sibilidad y que toma el promedio de las pérdidas (mejor métrica para tiempos de crisis financiera) en peores escenarios, es decir, cuando las pérdidas rebasaron ya el VaR. La pérdida esperada es la pérdida prevista (forward looking) que tendrá la institución en doce meses, calculada con el histórico de incumplimientos de más de 90 días. Gestiones eficientes de riesgo de crédito permiten incorporar estos cálculos en los resultados ya que se asocian a un costo más inherente al negocio de intermediación, ya que es lo que en este caso la Caja espera perder, dentro de un margen de seguridad, en promedio y que debería estar explicito en el cálculo de los ratios de rentabilidad, consiguiendo con esto una rentabilidad neta de las posibles pérdidas. El VaR y el CVaR son medidas que efectivamente corresponden a la mejor cuantificación del verdadero riesgo crediticio y cuya medición tienen que ser tomadas en cuenta por las instituciones para asignar los fondos propios constituidos por el capital y evitar quiebras por insolvencias ante escenarios desfavorables y no esperados.

Cuadro $\mathbf{N}^{\circ} 1$ : El siguiente gráfico reproduce una distribución de pérdidas ilustrativa por riesgo de crédito e identifica los parámetros de riesgo comentadas anteriormente. Estos cálculos se han realizado a cada una de las Cajas Municipales en el Perú al cierre del 2013 y 2014.

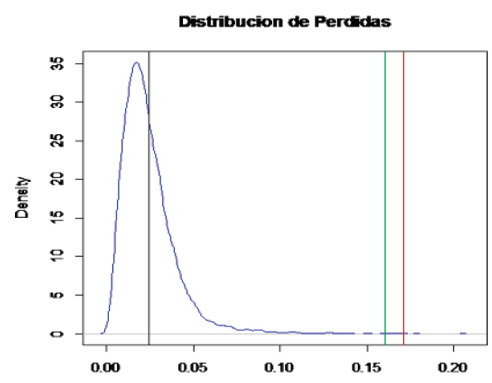

\begin{tabular}{|c|c|c|c|c|c|c|}
\hline Mínimo & $\begin{array}{c}1 \text { st } \\
\text { Qu. }\end{array}$ & Media & 3rd Qu. & $\begin{array}{c}\text { VaR } \\
(99.9 \%)\end{array}$ & $\begin{array}{c}\text { CVaR } \\
\text { (Expected S.) }\end{array}$ & Máximo \\
\hline $0,09 \%$ & $1,39 \%$ & $2,45 \%$ & $3,06 \%$ & $16,01 \%$ & $17,16 \%$ & $20,52 \%$ \\
\hline
\end{tabular}

En el eje vertical se identifica la densidad (\%) de la distribución de pérdidas y en el eje horizontal se muestra el porcentaje de pérdidas sobre la cartera vigente a comienzos del año analizado. Es una distribución asimétrica con cola ancha a la derecha representando con esto los altos montos de pérdidas pero con probabilidades bajas. La pérdidas esperada (línea negra) para este portafolio se ubica en $2.45 \%$, siendo la máxima pérdidas del orden del $20.52 \%$ del monto original de los créditos. La probabilidad que la pérdidas sea mayor al 16\% (línea verde) del valor total de la cartera es de $0.1 \%$, mientras que la pérdidas esperada por encima del VaR es del $17.16 \%$ (línea roja). La pérdidas inesperada (VaR-PE) se supone deberá estar cubierta con el capital de la institución, en este caso representa el 13.6\% de la cartera menor o igual a 90 días. 
Cuadro $\mathbf{N}^{\circ}$ 2: A continuación se presentan los resultados numéricos de las simulaciones ${ }^{16}$ por cada CMAC en ambos años del análisis.

\begin{tabular}{|c|c|c|c|c|c|c|c|c|}
\hline \multirow[b]{2}{*}{ CMACs } & \multicolumn{2}{|c|}{ Saldos Vigentes ( en Soles) } & \multicolumn{2}{|c|}{$\begin{array}{c}\text { Pérdidas } \\
\text { Esperada } \\
(\% \text { Saldos Vigentes })\end{array}$} & \multicolumn{2}{|c|}{$\begin{array}{c}\text { VaR } \\
\text { (\% Saldos } \\
\text { Vigentes })\end{array}$} & \multicolumn{2}{|c|}{$\begin{array}{c}\text { CVaR } \\
(\% \text { Saldos } \\
\text { Vigentes })\end{array}$} \\
\hline & 2012 & 2013 & 2013 & 2014 & 2013 & 2014 & 2013 & 2014 \\
\hline Arequipa & $2,392,101,843$ & $2,848,820,847$ & $4.03 \%$ & $4.75 \%$ & $11.29 \%$ & $13.39 \%$ & $12.13 \%$ & $14.10 \%$ \\
\hline Piura & $1,512,664,601$ & $1,866,116,870$ & $4.31 \%$ & $4.27 \%$ & $13.66 \%$ & $31.42 \%$ & $14.50 \%$ & $33.01 \%$ \\
\hline Huancayo & $1,023,235,732$ & $1,289,368,585$ & $2.69 \%$ & $2.53 \%$ & $11.98 \%$ & $12.39 \%$ & $13.08 \%$ & $12.89 \%$ \\
\hline Trujillo & $1,124,800,799$ & $1,276,280,828$ & $4.31 \%$ & $4.64 \%$ & $11.95 \%$ & $11.90 \%$ & $12.95 \%$ & $12.31 \%$ \\
\hline Cuzco & $1,016,508,881$ & $1,243,443,309$ & $2.41 \%$ & $2.62 \%$ & $10.99 \%$ & $17.46 \%$ & $11.95 \%$ & $18.12 \%$ \\
\hline Sullana & $1,128,323,136$ & $1,234,769,420$ & $5.85 \%$ & $6.07 \%$ & $14.99 \%$ & $18.94 \%$ & $16.17 \%$ & $19.54 \%$ \\
\hline Tacna & $591,686,978$ & $709,487,050$ & $3.59 \%$ & $5.49 \%$ & $13.85 \%$ & $13.81 \%$ & $14.69 \%$ & $14.53 \%$ \\
\hline Ica & $586,144,738$ & $673,392,319$ & $4.73 \%$ & $4.56 \%$ & $9.80 \%$ & $10.76 \%$ & $10.48 \%$ & $11.98 \%$ \\
\hline Maynas & $292,499,700$ & $308,578,552$ & $4.82 \%$ & $5.07 \%$ & $14.35 \%$ & $19.00 \%$ & $16.16 \%$ & $20.20 \%$ \\
\hline Paita & $200,946,393$ & $202,855,441$ & $6.74 \%$ & $5.09 \%$ & $17.91 \%$ & $19.04 \%$ & $19.23 \%$ & $19.74 \%$ \\
\hline Del Santa & $142,271,120$ & $162,186,063$ & $7.33 \%$ & $6.11 \%$ & $26.45 \%$ & $19.32 \%$ & $28.03 \%$ & $20.06 \%$ \\
\hline
\end{tabular}

Fuente propia.

El cuadro anterior nos indica los saldos vigentes así como las tres métricas de riesgo calculadas luego de doce meses como porcentaje de dichos saldos. En promedio el sector de Cajas Municipales tuvo un ligero incremento en términos de pérdidas esperadas anuales de +25 pbs, pasando de $4.15 \%$ a $4.40 \%$. El incremento del VaR promedio del sector pasó de $12.68 \%$ a $17.18 \%$, un incremento de +449 pbs. Un punto interesante de los resultados es que la pérdida esperada e inesperada carecen de una relación lineal exacta, lo cual se acentúa en el cálculo para el ejercicio 2013-2014 donde por ejemplo Caja Cuzco tiene una pérdidas esperada de $2.62 \%$ y una inesperada de $17.46 \%$ mientras que Cajas como Ica, Trujillo, Tacna y Arequipa tienen pérdidas esperadas por encima del $4 \%$ e inesperadas por debajo del 14\%. Esto se sustenta en el grado de granularidad de los portafolios. El Expected Shortfall (CVaR) debe ser siempre mayor al VaR de manera que, se estaría exigiendo mayor cantidad de reservas y no todas las instituciones poseen la solvencia para realizarlo desde un punto de vista regulatorio y de disciplina financiera. El CVaR medio para el sector incremento en $+246 p b s$ en un año (de $15.40 \%$ a $17.86 \%$ ), siete Cajas incrementaron esta medida en el análisis, siendo las principales Piura, Cuzco, Maynas y Sullana. El análisis de riesgo de crédito a través del cómputo de la distribución de pérdida indica las posibles pérdidas en un periodo determinado. Además, permite tomar en cuenta de manera confiable diversas características colectivas del grupo de individuos que conforman los portafolios, especialmente los efectos de la concentración y

16 Todos los cálculos del articulo se han llevado a cabo con R package. http://www.rproject.org/ 
granularidad en la cartera, características que los métodos estándar que utiliza el sistema financiero peruano pasan desapercibidas. A medida que los créditos de un portafolio se incrementan, el efecto de los créditos relativamente grandes (tickets grandes) en las colas de la distribución de pérdidas se va suavizando, es decir, el VaR o el capital económico tiende a disminuir y a converger a un portafolio granular, lo anterior condicionado a un nivel de riesgo dado. Por lo tanto, si la participación relativa de estos créditos con alta exposición respecto al resto de la cartera se incrementa, esto repercutiría en mayores niveles de pérdidas inesperadas que tendrían que estar cubiertas con mayores niveles de capital. En ese sentido, podemos decir que dado un nivel de riesgo del portafolio, la cantidad de créditos (granularidad) y el tamaño de éstos definitivamente importan, más aun en portafolios con un número pequeño de deudores.

\section{RENTABILIDAD Y SOLVENCIA}

Las medidas comúnmente conocidas de rentabilidad basadas en los estados financieros (no toman el riesgo incurrido), son la rentabilidad sobre los activos y la rentabilidad sobre el patrimonio. Estos no toman en consideración los riesgos inherentes a las actividades de intermediación lo que podría llevar a resultados divergentes de los reales, ya que no incorporan en su lo que se está arriesgando y que eventualmente podría configurarse en pérdidas. Utilizamos la rentabilidad esperada y ajustada al capital en riesgo (RARORAC) ya que por un lado el numerador nos muestra las ganancias tomando en cuenta las pérdidas esperadas, y en el denominador, el capital económico que refleja el apetito por riesgo de la institución. La fórmula es la siguiente:

$$
\operatorname{RARORAC}_{i}=\frac{U N_{i}-P E_{i}}{P I_{i}}
$$

Se utilizó la utilidad antes de impuestos del Estado de Resultados al cierre de 2014 de cada CMAC, añadiéndole el gasto de provisiones (equivale a pérdidas previstas). Suponemos para simplicidad del ejercicio que para el cálculo de la pérdida esperada e inesperada, el saldo vigente de los créditos en el activo de cada Caja tiene 90 o menos días de atraso.

Cuadro N`3: En el siguiente cuadro obtenemos el RAROC, que es una medida parcial del RARORAC, calculada como la rentabilidad ajustada por pérdidas esperada sobre el patrimonio efectivo, por ultimo tenemos el $\mathrm{ROE}^{5}$ contable que fue ajustado para poder compararlo con el RAROC, devolviéndole las provisiones y ajustándolo sobre el patrimonio efectivo. $\mathrm{p}^{17}$

\begin{tabular}{|l|c|c|c|c|c|c|}
\hline $\begin{array}{l}\text { CMACs } \\
\text { (Miles de S/.) }\end{array}$ & $\begin{array}{c}\text { Saldo } \\
\text { Vigente 2014 }\end{array}$ & $\begin{array}{c}\text { Pérdidas } \\
\text { Esperada }\end{array}$ & $\begin{array}{c}\text { Pérdidas } \\
\text { Inesperada }\end{array}$ & RARORAC & RAROC & ROE $^{17}$ \\
\hline Arequipa & $2,599,717$ & 123,487 & 224,616 & $50.8 \%$ & $21.5 \%$ & $19.1 \%$ \\
\hline
\end{tabular}

17 Utilidad antes del impuesto a la renta ajustada por el patrimonio efectivo. Cifras al cierre del 2014. 


\begin{tabular}{|l|c|c|c|c|c|c|}
\hline Piura & $1,826,750$ & 78,002 & 495,963 & $5.6 \%$ & $8.3 \%$ & $18.1 \%$ \\
\hline Huancayo & $2,599,717$ & 65,773 & 256,332 & $14.6 \%$ & $12.6 \%$ & $27.2 \%$ \\
\hline Trujillo & $1,281,847$ & 59,478 & 93,062 & $39.8 \%$ & $10.9 \%$ & $13.3 \%$ \\
\hline Cuzco & $1,405,075$ & 36,813 & 208,513 & $26.8 \%$ & $19.4 \%$ & $23.7 \%$ \\
\hline Sullana & $1,513,960$ & 91,897 & 194,847 & $8.2 \%$ & $6.3 \%$ & $18.5 \%$ \\
\hline Tacna & 604,623 & 33,194 & 50,305 & $26.0 \%$ & $11.2 \%$ & $11.3 \%$ \\
\hline Ica & 620,612 & 28,300 & 38,478 & $18.8 \%$ & $6.2 \%$ & $15.5 \%$ \\
\hline Maynas & 285,692 & 14,485 & 39,797 & $3.0 \%$ & $1.8 \%$ & $10.5 \%$ \\
\hline Paita & 155,311 & 7,905 & 21,666 & $-14.2 \%$ & $-8.9 \%$ & $0.6 \%$ \\
\hline Del Santa & 132,103 & 8,071 & 17,451 & $-2.4 \%$ & $-1.4 \%$ & $8.7 \%$ \\
\hline Total Cajas & $\mathbf{1 3 , 0 2 5 , 4 0 7}$ & $\mathbf{5 4 7 , 4 0 5}$ & $\mathbf{1 , 6 4 1 , 0 2 8}$ & $\mathbf{1 8 . 1 \%}$ & $\mathbf{1 2 . 3 \%}$ & $\mathbf{1 8 . 5 \%}$ \\
\hline
\end{tabular}

Fuente propia.

Como era de esperar los resultados muestran que las rentabilidades contables corregidas por la posibilidad de sucesos de impago medios e inesperados difieren entre sí, incluso llegan a ser hasta negativas para dos instituciones como son Caja Paita y Del Santa. Los resultados negativos indican que no hay generación de valor para dichas Cajas, la rentabilidad no estaría cubriendo el verdadero riesgo tomado. Tomar medidas de rentabilidad del tipo contable en instituciones financieras como son las Cajas, podrían estar sobreestimando las rentabilidades de éstas, diciendo poco de los riesgos asumidos para su obtención. El RAROC muestra rentabilidades menores al RARORAC ya que están calculadas sobre el patrimonio efectivo total (crédito, mercado y operacional) y no solo por la pérdida inesperada crediticia. El RARORAC a diferencia del RAROC ajusta el capital al verdadero riesgo crediticio (el que no se provisiona) que asume la entidad y que podría perderlo en búsqueda de obtener dichas ganancias. Existe un amplio consenso en el mundo sobre la necesidad de que las instituciones de crédito tengan la capacidad de cubrir sus pérdidas inesperadas con el capital propio para asegurar adecuados niveles de solvencia ante situaciones de estrés financiero.

Cuadro $\mathbf{N}^{\circ}$ 4: En el siguiente cuadro comparamos los resultados de las simulaciones con el ratio global de capital que presentaron las Cajas al cierre del 2014.

\begin{tabular}{|l|c|c|c|c|c|c|}
\hline $\begin{array}{c}\text { CMACs } \\
\text { (Miles de S/.) }\end{array}$ & $\begin{array}{c}\text { Patrimonio } \\
\text { Efectivo }\end{array}$ & $\begin{array}{c}\text { Ratio } \\
\text { Global } \\
\text { Simulado }\end{array}$ & $\begin{array}{c}\text { Ratio Global } \\
\text { SBS }\end{array}$ & $\begin{array}{c}\text { Requerimiento } \\
\text { de Capital } \\
\text { por Riesgo de } \\
\text { Crédito }\end{array}$ & $\begin{array}{c}\text { Ratio de } \\
\text { Capital por } \\
\text { Riesgo de } \\
\text { Crédito } \\
\text { (Simulación) }\end{array}$ & $\begin{array}{c}\text { Ratio Capital } \\
\text { SBS por } \\
\text { Riesgo de } \\
\text { Crédito }\end{array}$ \\
\hline Arequipa & 531,076 & $17.90 \%$ & $14.87 \%$ & 285,134 & $23.64 \%$ & $18.63 \%$ \\
\hline Cusco & 288,884 & $12.26 \%$ & $14.08 \%$ & 177,996 & $13.86 \%$ & $16.23 \%$ \\
\hline Del Santa & 29,127 & $13.22 \%$ & $12.88 \%$ & 18,045 & $16.69 \%$ & $16.14 \%$ \\
\hline Huancayo & 297,607 & $10.34 \%$ & $15.64 \%$ & 158,927 & $11.61 \%$ & $18.73 \%$ \\
\hline Ica & 117,264 & $21.61 \%$ & $14.38 \%$ & 65,758 & $30.47 \%$ & $17.83 \%$ \\
\hline Maynas & 65,136 & $13.54 \%$ & $15.56 \%$ & 33,554 & $16.37 \%$ & $19.41 \%$ \\
\hline Paita & 34,716 & $13.04 \%$ & $14.07 \%$ & 19,734 & $16.02 \%$ & $17.59 \%$ \\
\hline
\end{tabular}




\begin{tabular}{|l|c|c|c|c|c|c|}
\hline Piura & 333,626 & $6.13 \%$ & $13.35 \%$ & 201,711 & $6.73 \%$ & $16.54 \%$ \\
\hline Sullana & 255,490 & $11.08 \%$ & $11.79 \%$ & 180,742 & $13.12 \%$ & $14.14 \%$ \\
\hline Tacna & 117,197 & $18.15 \%$ & $15.41 \%$ & 61,734 & $23.31 \%$ & $18.98 \%$ \\
\hline Trujillo & 341,198 & $26.26 \%$ & $17.41 \%$ & 159,088 & $36.65 \%$ & $21.45 \%$ \\
\hline Total Cajas & $\mathbf{2 , 4 1 1 , 3 2 0}$ & $\mathbf{1 3 . 5 5} \%$ & $\mathbf{1 4 . 5 1 \%}$ & $\mathbf{1 , 3 6 2 , 4 2 2}$ & $\mathbf{1 6 . 2 9 \%}$ & $\mathbf{1 7 . 7 0} \%$ \\
\hline
\end{tabular}

Fuente propia.

Observamos divergencia entre los ratios de capital regulatorios con los hallados por la simulación. Por un lado tenemos el ratio global simulado que incorpora el capital inesperado calculado así como el requerimiento de capital por los otros dos riesgos (mercado y operacional). El ratio global SBS para el sector se ubica por encima del simulado, esto por efectos de mayores exigencias de capital por riesgo crediticio que muestra el ejercicio a través de las pérdidas inesperadas. Particularmente hay resultados mixtos, más de la mitad de las Cajas mostraron ratios simulados menores a los regulatorios, las mayores diferencias se dan en las Cajas Piura y Huancayo que tienen ratios SBS de $13.35 \%$ y $15.64 \%$ respectivamente pero solo llegan a $6.13 \%$ y a $10.34 \%$ en los simulados, esto debido a que las pérdidas inesperadas en el caso de Caja Piura sumaron S/. 496 millones mientras que el requerimiento por riesgo de crédito fue de tan solo S/. 202 millones, es decir 2.45 veces más que el asignado regulatoriamente, Huancayo muestra 1.6 veces mayores pérdidas inesperadas que el capital regulatorio asignado. En el otro extremo se ubican las Cajas de Trujillo, Ica, Arequipa y Tacna cuyas pérdidas inesperadas resultaron menores al capital propio asignado para cubrirlas, con lo cual podríamos concluir que muestran mejores niveles de respaldo ante eventos no esperados. Las pérdidas inesperadas son las que le dan formas distintas a las distribuciones de pérdidas, haciendo las colas más anchas para aquellas instituciones que tienen en sus portfolios mayor dispersión en los tamaños de sus créditos. La concentración (no granularidad) en las exposiciones de un portfolio de crédito es un factor importante en la medición del riesgo crediticio. Existen dos factores que impactan en la concentración por cliente (name concentration) debido a la imperfecta diversificación del riesgo idiosincrático, el primero es causado debido a que el portafolio consta de un número pequeño de préstamos, el segundo, se debe a que en un portafolio pueden haber altas exposiciones en términos relativos en algunos créditos particulares. Para evitar problemas de solvencia ante deterioros de actividad económica es importante que también las instituciones financieras monitoreen y controlen permanentemente el apetito (en términos de capital económico) del portafolio y de cada una de sus líneas de negocio a través de límites que acoten la dispersión del tamaño de sus créditos, mas aun en instituciones financieras con menores accesos al mercado de capitales como son la gran mayoría de empresas dedicadas a las micro finanzas. 


\section{CONCLUSIONES}

Según los cálculos del ejercicio, 6 de las 11 Cajas tendrían ratios de capital por riesgo de crédito subestimados, donde las mayores discrepancias están en la Caja Piura y Caja Huancayo, donde sus niveles de solvencia regulatoria divergirían mucho con respecto a la empírica dados los supuestos utilizados en el análisis. Medidas de rentabilidad contables deberían ser ajustadas al riesgo para sensibilizar efectivamente lo que la institución está arriesgando en búsqueda de dicha rentabilidad y las pérdidas esperadas ajusten el margen obteniendo una medida neta de pérdidas probables como gasto natural del negocio y asimismo ajustadas al capital aparcado y necesario para cubrir pérdidas inesperadas, conservando un nivel sólido de solvencia. De este modo, se asegura estar valorando con precisión los recursos utilizados en la inversión. Cajas que no estarían agregando valor a los accionistas son las Cajas Paita y Del Santa. Como se ha podido confirmar tras los resultados del análisis, el tamaño sí importa. Desde el punto de vista de solvencia, los requisitos de capital regulatorio también deberían de incorporar la heterogeneidad entre instituciones financieras en función a indicadores de granularidad de la cartera crediticia. Cálculos continuos del CVaR permitirían el monitoreo de una medida de estrés del portafolio de créditos y que pondrían en alerta a las Cajas ante posibles escenarios desfavorables de crisis financiera.

\section{REFERENCIAS BIBLIOGRÁFICAS}

1. Mora Valencia, Andrés. (2010). Revista Civilizar.

2. Adasme Osvaldo, Majnoni Giovanni, Uribe Myriam. SBIF-Chile (2006). Calibración del Riesgo de Crédito en los Países Emergentes: La experiencia de Chile. Federación de Cajas Municipales de Ahorro y Crédito Perú 2012.

3. Tarca Silvio. (2014). Regulatory Capital Modelling for Credit Risk. School of Mathematics and Statistics. University of Sidney.

\section{SOFTWARE UTILIZADO}

- http://www.r-project.org/

Una propuesta de CreditMetrics y Expected Shortfall para Riesgo de Crédito. 